Relations industrielles

Industrial Relations

\title{
Les cartels et l'arbitrage des prix
}

\section{Maurice Lamontagne}

Volume 5, numéro 7, avril 1950

URI : https://id.erudit.org/iderudit/1023359ar

DOI : https://doi.org/10.7202/1023359ar

Aller au sommaire du numéro

Éditeur(s)

Département des relations industrielles de l'Université Laval

ISSN

0034-379X (imprimé)

1703-8138 (numérique)

Découvrir la revue

Citer cet article

Lamontagne, M. (1950). Les cartels et l'arbitrage des prix. Relations industrielles / Industrial Relations, 5(7), 63-66. https://doi.org/10.7202/1023359ar

Tous droits réservés (C Département des relations industrielles de l’Université Laval, 1950
Ce document est protégé par la loi sur le droit d'auteur. L'utilisation des services d'Érudit (y compris la reproduction) est assujettie à sa politique d'utilisation que vous pouvez consulter en ligne.

https://apropos.erudit.org/fr/usagers/politique-dutilisation/ 
Il est facile de constater que l'Episcopat du Québec, après le Pape lui-même, n'est pas le seul à préconiser des réformes dans la structure de l'entreprise. La même remarque pourrait s'appliquer d beaucoup d'autres passages de la lettre sur "le problème ouvrier en regard de la doctrine sociale de l'Eglise». En plus des directives de ce genre qui ont déjà été faites en d'autres pays, ce document épiscopal contient un grand nombre de recommandations qui s'appliquent tout spécialement a la province de Québec. Ces deux aspects de la lettre collective témoignent à la fois de l'unité d'enseignement de la Hiérarchie à travers le monde et de son souci d'adaptation selon les milieux et les époques.

"Le problème ouvrier en regard de la doctrine sociale de l'Eglise » est un magistral travail qui, en plus de stimuler les apôtres sociaux, fait progresser très considérablement la science sociale chrétienne. Il y a là deux motifs plus que suffisants pour réjouir la Faculté des sciences sociales, le Département des relations industrielles et le Bulletin des relations industrielles.

LA RÉDAction

\title{
LES CARTELS ET L'ARBITRAGE DES PRIX
}

\author{
Maunice Lamontagne
}

\section{Monopole, trust et cartel}

Des événements récents ont remis le problème des cartels plus que jamais à l'ordre du jour. Pour être en mesure de mieux le définir, il faut bien préciser les différences qui existent entre cartel, trust et monopole et qui sont souvent négligées. Le monopole correspond à la situation où il n'y a qu'un seul vendeur sur le marché d'un produit, lequel se trouve ainsi en position de fixer à sa guise le volume de la production et le niveau des prix. Il y a deux façons d'organiser un monopole. La première, c'est le trust où une seule entreprise détient le monopole. Par exemple, la Compagnie Canadian Industries Limited est un trust, car elle possède à elle seule un monopole sur plusieurs produits chimiques. Le cartel constitue la seconde façon d'organiser un monopole. Il est formé par une association de plusieurs pro. ducteurs d'un même produit qui s'entendent pour poursuivre une même politique de prix et de production. L'industrie de la pulpe et du papier, par exemple, était, jusqu'à récemment du moins, organisée en cartel. Canadian Industries Limited, qui possède le monopole de plusieurs produits au Canada, fait partie, conjointement avec Imperial Chemical d'Angleterre et Dupont de Nemours des Etats-Unis, du cartel international de l'industrie chimique.

\section{Modes de formation des cartels}

Depuis vingt ans surtout, le mouvement des cartels s'est intensifié au Canada comme ailleurs. De façon générale, on peut dire qu'il existe un cartel dans chaque industrie où le nombre d'entreprises est restreint. Il est donc possible de se faire une idée de l'extension du mouvement en constatant l'importance que possède un nombre restreint d'entreprises à l'intérieur de leur industrie respective.

Quant au nombre exact de cartels existant présentement il est absolument impossible de l'évaluer. Cette impossibilité est attribuable aux différentes méthodes auxquelles on a recours pour organiser un cartel.

En effet, l'entente entre les producteurs qui est à l'origine du cartel peut être formelle ou implicite. A son tour, l'entente formelle peut être écrite ou verbale. Il y a entente implicite lorsque, par exemple, sans qu'il y ait eu rencontre préalable, les plus petits producteurs dans une industrie ne font que suivre la politique décidée par la ou les plus grandes entreprises et qu'imiter leur action. Au cours d'une période encore récente, il n'existait aucune entente formelle et précise entre les différents producteurs d'acier aux EtatsUnis. Le cartel de l'acier n'en était pas moins efficace, car toutes les entreprises acceptaient d'avance et appliquaient elles aussi les décisions prises par United States Steel Corporation concernant les prix et le volume de la production. Les méthodes de l'entente verbale et de l'entente implicite sont certainement les plus en vogue aujourd'hui, et c'est précisément pour cette raison qu'il n'est pas possible d'estimer, même approximativement le nombre des cartels.

\section{Cartels et législation}

Les gouvernements ne pouvaient pas rester indifférents à la rapide multiplication des cartels, car leurs conséquences normales, qui consistent principalement à restreindre systématiquement la production et à élever indûment les prix, sont 
absolument incompatibles avec les exigences du bien-être général. En effet, les cartels ont des effets néfastes dans tous les secteurs de l'économie: ils engendrent le chômage, ils contribuent à élever indûment le coût de la vie, ils entraînent une distribution injuste du revenu national en assurant des profits trop élevés; ils constituent la négation même de l'économie de concurrence et ils sont en flagrante contradiction avec la doctrine capitaliste.

Au Canada, la Loi des combines condamne les ententes qui nuisent à l'intérêt public. Les entreprises qui font de telles ententes sont susceptibles d'être poursuivies et d'être condamnées à payer des amendes. Cette loi tombe sous la juridiction du gouvernement fédéral et il appartient au commissaire en charge d'appliquer la loi d'entreprendre les enquêtes nécessaires pour prouver l'existence des ententes. Toutefois, les poursuites devant les tribunaux relèvent des gouvernements provinciaux.

Il ne faut pas oublier que la loi ne défend pas les cartels en eux-mêmes. Pour qu'elle s'applique il faut prouver non seulement qu'il y a eu entente, mais également que cette entente est contraire à l'intérêt public. Les limitations de cette loi sont nombreuses et méritent d'être soulignées.

1. En premier lieu, la loi ne couvre pas le cas des trusts. Il est assez paradoxal de constater qu'elle empêche un groupe d'entreprises de s'entendre pour agir contre l'intérêt public, mais qu'elle ne défend pas à une compagnie d'acquérir des entreprises concurrentes en vue de constituer un monopole et d'exploiter le public. C'est le cas de la compagnie «Famous Players».

2. En ce qui regarde les cartels eux-mêmes, la loi exige d'abord qu'une preuve directe de l'existence d'une entente soit établie, car les accusés sont présumés non coupables. Or il est pratiquement impossible de faire cette preuve s'il n'y a pas d'entente écrite entre les entreprises. Donc tous les cartels qui n'ont pas d'entente écrite, et c'est le très grand nombre, peuvent se soustraire à la loi assez facilement.

3. Non seulement l'entente écrite doit exister, mais encore il faut que les officiers publics en apprennent l'existence et puissent la découvrir. Or ces deux conditions ne sont pas facilement remplies, car les membres d'un cartel se vantent rarement d'avoir signé une entente illégale.

4. Une fois l'existence de l'entente directement prouvée, la loi exige en plus que les officiers publics soient en mesure de démontrer, à la satisfaction du tribunal, que la dite entente est contraire à l'intérêt général. Cette deuxième exigen- ce de la loi est probablement plus rigoureuse que la première, car elle laisse libre cours à toutes les interprétations subjectives.

5. Lorsque, à la suite des procédures longues et difficiles qui sont exigées, le tribunal trouve la preuve suffisante, il impose une amende aux membres du cartel et ordonne la cessation de l'entente. Les amendes imposées sont ridiculement basses puisqu'elles ne peuvent dépasser $\$ 10,000$ pour les individus et $\$ 25,000$ pour les compagnies. Ainsi, les sommes à débourser à la suite de la condamnation sont bien inférieures aux avantages retirés de l'entente. Quant à l'ordre de cesser l'entente, il est rarement exécuté. On fait ordinairement disparaître les écrits, mais le cartel luimême demeure aussi efficace qu'auparavant grâce à une entente verbale ou implicite.

6. En plus des limitations de la loi ellemême, on doit ajouter que les différents gouvernements n'ont pas favorisé son application et qu'ils n'ont pas donné aux administrateurs ni l'encouragement ni le personnel nécessaires à une action efficace.

La présente loi, à laquelle on a apporté quelques amendements d'importance secondaire, est en vigueur depuis 1923. Au cours de cette période, 20 enquêtes ont été menées; 9 cartels ont été découverts, dont 4 ont probablement été dissous par suite des condamnations. Quand on songe qu'au cours de cette même période, le mouvement des cartels et des trusts s'est considérablement intensifié au Canada, on ne peut s'empêcher de reconnaître que la présente loi est bien loin d'offrir une solution satisfaisante au problème du monopole.

Assez paradoxalement, il semble qu'elle soit trop exigeante et qu'elle veuille trop accomplir. En effet, elle se donne comme objectif d'empêcher la naissance ou d'entraîner la disparition des cartels contraires à l'intérêt public. La croyance naïve à la possibilité d'un retour et du maintien d'un système de concurrence pure constitue le vice fondamental de la conception sur laquelle elle repose.

Et pourtant, la seule façon pratique et efficace de faire disparaître un cartel consiste à nationaliser les entreprises qui en font partie. En effet, tant qu'elles subsistent, par quel système de surveillance réussira-t-on à empêcher que leurs dirigeants communiquent entre eux et s'entendent d'une façon ou d'une autre afin de sauvegarder leurs intérêts qui coïncident rarement avec ceux du public ? 
Toutefois cette solution radicale n'est pas compatible avec la structure du capitalisme et son application signifierait un changement de régime économique. Il ne faut pas oublier que l'évolution normale du système capitaliste mène presque inévitablement au trust et au cartel. Une loi qui condamne cette évolution peut satisfaire une foule de gens qui se contentent d'une fausse sécurité, mais elle devient le plus souvent inopérante, car la superstructure juridique ne saurait contrecarrer effectivement une tendance fondamentale de la structure économique. Soyons donc réalistes et reconnaissons, même sur le plan juridique, qu'en acceptant le capitalisme, nous admettons également l'existence des trusts et des cartels.

\section{Vers une solution réaliste : l'arbitrage des prix}

Mais alors, puisqu'il est impossible de faire disparaître ces tentatives de monopoliser le marché, est-ce à dire qu'il faille cesser de s'y opposer? Bien au contraire. Mais alors il faut trouver une solution dont on peut espérer certains résultats. Puisqu'il est très difficile d'empêcher l'existence des trusts et des cartels à l'intérieur d'une structure capitaliste, cherchons au moins à éviter leurs effets les plus néfastes sur les prix et la production.

La solution qui s'offre n'est pas nouvelle, puisqu'elle est déjà appliquée à un domaine très important de l'économie : le marché du travail. L'avènement des unions ouvrières a posé des problèmes nouveaux à une société économique où les salaires et les prix étaient censés être fixés par le mécanisme de la concurrence. En effet, les syndicats ouvriers tentent de monopoliser l'offre de main-d'oeuvre, afin de permettre aux travailleurs de transiger sur un pied d'égalité avec leur employeur. Laissées à elles-mêmes, les unions ouvrières auraient peut-être été en mesure, en certaines circonstances, de hausser indûment le niveau des salaires.

On a voulu protéger l'employeur, qui est le consommateur de travail, en conditionnant l'exercice du droit de grève par l'arbitrage obligatoire des salaires. Le régime de l'arbitrage obligatoire n'a pas fait disparaître aucun droit fondamental des ouvriers, puisque la sentence arbitrale n'est pas exécutoire. A l'intérieur de ce système, les travailleurs ont toujours le droit de fixer le prix de leur travail et de faire la grève en vue d'obtenir ce qu'ils demandent. Toutefois, le régime de l'arbitrage obligatoire contribue à ralentir la montée des salaires. D'abord il a pour conséquence de réduire le nombre et l'importance des demandes d'augmentation de salaire en forçant les unions ouvrières à justifier leurs exigences devant un tribunal. De plus, il est prouvé que dans des conditions normales, les unions ouvrières ne peuvent pas gagner beaucoup en ne se conformant pas à une sentence arbitrale, surtout lorsque celle-ci peut être considérée comme juste. Il résulte donc qu'elle est accejtée dans la plupart des cas, même si elle n'accorde qu'une partie des demandes des travailleurs. On peut donc conclure que l'arbitrage obligatoire a au moins empêché les augmentations injustifiées de salaire.

Cette technique particulière pourrait être appliquée également dans le domaine des prix. L'arbitrage obligatoire des prix contribuerait au moins autant que celui des salaires à éviter les augmentations injustifiées. Le seul fait d'obliger les entreprises à démontrer la nécessité d'une hausse de prix devant un tribunal aurait d'abord pour effet d'éliminer les augmentations que les industriels ne penseraient pas pouvoir justifier publiquement. De plus, même si la sentence arbitrale n'est pas exécutoire, les entreprises devraient s'y conformer plus ou moins, car elles seraient mal placées du point de vue de l'opinion publique pour imposer une hausse de prix qui viendrait d'être déclarée injustifiée. Enfin, au cas où elles ne s'y conformeraient pas, les unions ouvrières seraient en mesure d'exiger une part de la hausse non justifiée, ce qui constituerait une compensation pour la majorité des consommateurs.

L'arbitrage obligatoire peut donc atteindre toute la structure des prix. Il s'attaque directement aux conséquences néfastes des cartels et des trusts sans requérir au préalable l'établissement de la preuve de leur existence. Ces tentatives de monopole ont pour objectif de restreindre la production afin de maintenir un niveau de prix trop élevé. L'arbitrage obligatoire, en contribuant à abaisser les prix, entraînera indirectement une augmentation de la production, car les industriels devront adapter leur échelle d'opération à la nouvelle situation. En d'autres termes, l'arbitrage obligatoire, bien que ne s'attaquant pas directement à l'existence des cartels et des trusts, fait disparaître l'objectif en vue duquel ils sont organisés, ce qui est probablement le meilleur moyen d'amener leur disparition.

De plus, il n'est pas nécessaire qu'une entreprise constitue un trust ou fasse partie d'un cartel pour réussir à imposer des prix exagérés. En d'autres termes, il n'est pas essentiel de posséder un monopole parfait pour contrôler le marché et obtenir des prix injustifiés. Aucune législation destinée à combattre les cartels et les trusts ne peut remédier à cette situation; seul l'arbitrage 
obligatoire des prix peut apporter une solution satisfaisante.

Enfin l'application d'un tel système entraînerait un rendement plus élevé et une meilleure utilisation des ressources productives de la nation. D'abord, en contribuant à abaisser les prix, il stimulerait la production, ce qui signifierait une échelle d'opérations plus efficace et une diminution de la capacité de production inutilisée. En second lieu, il faut noter que dans plusieurs cas, une augmentation des prix ou leur maintien à un niveau élevé ne peut se motiver que par une mauvaise administration de l'entreprise qui ne s'efforce pas d'obtenir le meilleur rendement possible des facteurs de production. Le système actuel, par lequel le manque de compétence ou l'insouciance des administrateurs entraîne des hausses de prix, constitue une prime à l'inefficacité dont les frais sont supportés par l'ensemble de la société. Avec l'arbitrage des prix, les entreprises devraient tôt ou tard améliorer leur système administratif ou bien assumer les frais attribuables à son imperfection.

En somme, cette réforme ne s'inspire pas, comme la présente loi des combines, de la croyance naïve au retour à un système de concurrence pure qui n'a jamais existé et qui ne peut pas être réalisé. Elle prend l'organisation industrielle telle qu'elle est, avec ses tendances monopolistiques, et elle s'efforce de la réorienter de façon à ce qu'elle produise les mêmes avantages que ceux qu'offre le système de la concurrence.

L'arbitrage obligatoire des prix n'est pas nouveau. Jusqu'ici, cependant, il a été appliqué à un secteur limité de l'économie, qu'on appelle les utilités publiques. Ce groupe comprend les différents modes de transport, le téléphone et l'électricité. De plus, dans le système présentement en vigueur, la sentence arbitrale ou, si l'on veut, les décisions des administrateurs chargés de représenter les pouvoirs publics sont exécutoires.

Ce qu'il y a de nouveau dans la présente proposition c'est d'étendre l'application de ce principe de l'arbitrage aux principaux secteurs de l'économie où elle semble désirable, sans toutefois rendre la décision arbitrale exécutoire.

Cette réforme semble amplement se justifier par les heureuses conséquences qu'on peut en espérer. De plus, elle ne présente pas d'inconvénients sérieux. Sur le plan administratif, par exemple, elle offre beaucoup moins de complications que le contrôle des prix, lequel a pourtant fonctionné effectivement au cours du conflit.

D'autre part, on pourrait prétendre que l'arbitrage obligatoire des prix restreint les prérogatives de l'entreprise privée. Toutefois, si on regarde de près, on constate qu'il garantit beaucoup plus qu'il ne limite la liberté d'entreprise. Il exige tout simplement que les industriels rendent compte de leurs actes à l'autorité publique. Sans diminuer la liberté de l'entreprise, il lui rappelle ses responsabilités sociales. Et, de ce point de vue, il peut contribuer à protéger l'entreprise privée contre elle-même, car si elle ne devient pas plus sociale, elle risque tôt ou tard d'être socialisée. L'arbitrage des prix aura certainement pour conséquence de rendre plus difficile l'augmentation injustifiée des prix, mais on ne saurait prétendre que la liberté d'entreprise implique même le droit de maintenir les prix à un niveau exagéré et d'exploiter systématiquement les consommateurs.

D'ailleurs, même si, à la suite de l'application de cette mesure, les industriels avaient l'impression d'être moins libres, leur condition ne serait certainement pas pire que celle de leurs employés. A l'intérieur d'une démocratie vraiment saine, comment pourrait-on s'opposer à l'arbitrage des prix des marchandises, alors que le prix du travail, c'est-à-dire le revenu de la grande majorité des consommateurs, est déjà soumis à ce système ? L'arbitrage effectif des prix constitue un des fondements de la démocratie économique et une méthode efficace de lutte contre les cartels, les trusts et contre toutes les tendances monopolistiques présentes.

\section{Avez-vous besoin d'un agent de relations industrielles?}

Nous pouvons vous mettre en relation avec des diplômés spécialisés en ce domaine et dont les connaissances théoriques s'allient à une expérience pratique.

Pour toute information, prière de communiquer avec Jean-H. Gagné, secrétaire du Département des relations industrielles, Faculté des sciences sociales, Université Laval, 2, rue de l'Université, Québec. 